\title{
Geneviève MADORE
}

\section{Balzac, homme politique légitimiste et visionnaire}

«Balzac était visionnaire, nous l'avons fait réaliste, et c'était partir à la recherche d'un autre Balzac. Balzac était de droite, nous l'avons mis à gauche, est réapparu l'autre Balzac», ironise Franc Schuerewegen (2004, p. 16) en parlant du caractère insaisissable de l'auteur de La Comédie humaine. La porosité de la frontière séparant Balzac et son œuvre rend d'autant plus difficile la tâche de trancher entre les opinions énoncées dans ses articles, ses romans, ses préfaces et sa correspondance. Au-delà des flottements, des contradictions et des ambiguités, on retient que Balzac, avec Musset et Chateaubriand, fut l'un des détracteurs du « juste milieu». Sa prolifique relation avec la presse, au cours des années 1830, même si elle était due à ses déboires dans le monde de l'édition parisienne, prouve hors de tout doute qu'il était un homme de conviction. Souvent cinglant et avec une verve qu'on ne retrouve pas dans ses romans, il va de règlements de comptes en justifications, attaquant ses détracteurs du milieu de l'édition, mais aussi les tendances et les modes qui frappent de plein fouet la société française au lendemain des Trois Glorieuses. Attentif à l'évolution des mœurs, Balzac fut aussi en contact étroit avec la politique tout au long de sa carrière d'écrivain.

Paul Métadier, dans son Balzac homme politique, propose de se pencher à nouveau sur la pensée politique de Balzac: «Il ne fut pas homme d'État puisque le système censitaire ne lui permit pas d'en briguer les fonctions lorsqu'il était dans la force de l'âge, mais il a marqué son époque par la puissance des témoignages qu'exprime sa peinture. » (p. 18) Si Balzac n'a pas eu de carrière politique comme ce fut le cas de Chateaubriand et de Lamartine, il ne resta pas indifférent devant les effets de la Révolution de Juillet, dont il souffrit en tant qu'écrivain. C'est cet «homme politique » que Paul Métadier propose de décrire dans son dernier livre: "Homme politique [...] par la part qu'il prendra avec ses écrits par cette lente maturation d'une pensée 
Geneviève MADORE, «Balzac, homme politique légitimiste et visionnaire », @nalyses, automne 2007

politique qui dominera son siècle, et le nôtre, bien sûr celui qui vient de s'achever. » (p. 13) En s'engageant ainsi sur un terrain très vaste et maintes fois ratissé, Métadier fait preuve d'audace. Heureusement, un consensus sur la pensée politique de Balzac n'a pas encore été établi et plusieurs questions restent en suspens. Par exemple, doit-on attribuer à Balzac la parenté de l'article « Le droit d'aînesse » (1824), où il fustige le mode de division de la propriété établi par la révolution de 1789? Que faire d'un roman comme Les Chouans (1829), qui encense tour à tour républicains et royalistes? Balzac est-il réactionnaire ou visionnaire? Une chose est sûre : l'auteur de La Comédie bumaine n'aura de cesse, à partir de 1830, de décrier la «médiocrité » qui règne au sein de la société française louis-philipparde et de ses institutions.

Dès le premier chapitre («L'ambition»), qui tient lieu d'introduction, Métadier fixe clairement les limites d'ordre méthodologique de sa démarche : son ouvrage ne renouvellera pas le travail titanesque déjà fait par un Pierre Barbéris ou un Bernard Guyon et, ajouterions-nous, par leurs nombreux successeurs, notamment les Max Andréoli, André Vanoncini, José-Luis Diaz, Alexandre Courteix, pour ne nommer que quelques-uns de ceux qui ont modernisé la critique balzacienne. Après l'apologie d'usage - lieu commun des études balzaciennes : que dire qui n'ait pas encore été dit? Sous quel angle n'a-t-on pas encore abordé l'œuvre de Balzac? - Métadier précise qu'il veut faire la lumière sur les aspects qui auraient été oubliés par la critique : «[...] et c'est là, la justification de notre propos, beaucoup d'événements contemporains [à Balzac] sont restés dans la pénombre » (p. 12).

L'objectif second de Métadier est de taille: écarter les stéréotypes gravitant autour de la personne de l'écrivain en robe de bure. Dans cette optique, il refuse de réduire la pensée politique de Balzac au passage le plus cité - et souvent galvaudé — de l'«Avantpropos »: "J'écris à la lueur de deux Vérités éternelles : la Religion, la Monarchie, deux nécessités que les événements contemporains proclament, et vers lesquelles tout écrivain de bon sens doit essayer de ramener notre pays. » (1976, p. 13) Le passage suivant, tiré de l'article inédit «Du gouvernement moderne », prouve d'ailleurs la pertinence de l'angle privilégié par Métadier, qui choisit de creuser la question du 
Geneviève MADORE, «Balzac, homme politique légitimiste et visionnaire », @nalyses, automne 2007

Balzac réactionnaire: «La classe moyenne avait soif d'égalité. Louis XVIII eût à jamais sauvé la branche aînée, s'il eût osé continuer Robespierre, moins l'échafaud. En supprimant hardiment toute noblesse, hors la Chambre des Pairs, il eût fait respirer à la classe moyenne un air libre.» (1956, p. 100) De Karl Marx, qui voyait en Balzac un révolutionnaire, à Philippe Muray, qui parle de la prise d'otage de Balzac par les marxistes (voir 1984, p. 369-382), plusieurs se sont prononcés sur le sens à donner à la profession de foi politique balzacienne. Or, comme le précise Métadier avec raison, malgré sa peur des masses jamais démentie, malgré son intérêt pour les contrerévolutionnaires comme Louis de Bonald et Joseph de Maistre, Balzac ne peut être enfermé dans le clan des réactionnaires: «[...] ces apparences ont retenu l'attention au-delà d'une pensée insuffisamment approfondie qu'il faut éclairer par le contexte, historique, économique et affectif des vingt années de sa maturité » (p. 14). Au plus fort de la production balzacienne, ce sont plus les débuts timides de l'ère industrielle en France et l'essor inexorable du capitalisme opéré par la bourgeoisie que la lutte ouvrière qui ont servi de toile de fond.

«Écrit d'un trait», comme le dit son préfacier (Jean Tulard, de l'Institut), Balzac homme politique mérite de faire partie « des ouvrages de référence de toute bibliothèque balzacienne » (p. 10). À la fin du livre, on trouve ce petit prologue signé, cette fois, par l'auteur lui-même, qui offre un tout autre point de vue sur l'ouvrage : «Il vaut mieux, dit-on, parler de mémoire plutôt que lire une conférence. Certes, des omissions et des imprécisions sont inévitables lorsque l'orateur est pris par son sujet. [...] C'est dans cet esprit que ce petit livre a été écrit. Aussi, dans l'immensité des études sur Balzac et son œuvre, la prétention de cet ouvrage est-elle modeste » (p. 179). Ces témoignages, s'ils sont contradictoires, n'en sont pas moins tous les deux vrais. Si Balzac homme politique ne renouvelle pas le genre, il constitue une bonne préparation aux travaux sur la période postrévolutionnaire d'Éric Hobsbawm ou sur ceux portant sur tout le $\mathrm{XIX}^{\mathrm{e}}$ siècle (Philippe Muray et René Rémond). Ancien conservateur du Musée Balzac à Saché et membre de l'Institut, l'auteur, surtout connu pour ses travaux de biographe (Balzac au petit matin, La Palatine, 1964) et Saché dans la vie et l'cuvre de Balzac, Calmann-Lévy, 1950), adopte ici une approche aux 
Geneviève MADORE, «Balzac, homme politique légitimiste et visionnaire », @nalyses, automne 2007

confluents de la biographie, de la politique et de l'histoire pour « étudier Balzac à la lumière de son temps » (p. 12).

\section{Un survol éclectique de la France sous la monarchie de Juillet}

Intéressé surtout par l'époque la plus productive de Balzac, soit les décennies 1830 et 1840, Métadier effectue une description de «La France sous Louis-Philippe », titre du deuxième chapitre : "Le monde de Balzac est celui de Louis-Philippe. Fils du régicide, Louis-Philippe a validé à Jemmapes son titre de roi-citoyen» (p. 39). Son esquisse politique, économique et sociale des années 1830 à 1848, époque de transition marquée par les insurrections et les tentatives de coup d'État, est plus anecdotique que conceptuelle. Les courants idéologiques n'y sont pas abordés, mais plutôt leurs manifestations concrètes: «La valse des gouvernements se poursuivit par un gouvernement Soult avec Thiers, puis un autre avec Broglie et Guizot, puis à nouveau Soult sans Thiers ni Guizot. » (p. 50) Y sont tout justes mentionnés la chute de la monarchie, le développement de l'industrie (chemin de fer et charbon) et l'avancement de la médecine. Se faisant conteur plus qu'historien, Métadier évoque les rencontres entre Balzac et Vidocq, ancien bagnard et informateur du gouvernement, qui lui fournit plusieurs informations sur les souterrains de la ville de Paris et lui inspira le thème des sociétés secrètes. Certaines formules heureuses font une synthèse efficace de l'atmosphère sous la Monarchie de Juillet : "James Rothschild a trente-huit ans. Il est le roi de Paris.» (p. 26), «La puissance de l'argent est au centre des bouleversements économiques en gestation. » (p. 26) Si Métadier résume de manière très succincte la pensée de Joseph de Maistre et de Louis de Bonald et, passages obligés, celles de Chateaubriand et de Tocqueville, il s'attarde davantage sur Saint-Simon, Lamennais et Pierre Leroux. La pièce de résistance du chapitre, également évoquée dans les chapitres ultérieurs, est le difficile tournant de 1840, qui "préfigure l'effondrement de la Monarchie de Juillet» (p. 53), marquée par des émeutes. Celles-ci rappellent au gouvernement la misère du peuple et le forcent à adopter, en 1841, la loi Montalembert, première loi sociale limitant le nombre d'heures de travail pour les enfants. 
Geneviève MADORE, «Balzac, homme politique légitimiste et visionnaire », @nalyses, automne 2007

En deuxième partie de chapitre, Métadier décrit Louis-Philippe comme un bon père, mais comme un roi manquant d'autorité, victime de cette période d'instabilité. Successeur de l'autoritaire Charles X, Louis-Philippe, roi du compromis, fit malgré tout montre de trop de rigidité pour s'attirer la sympathie populaire. Métadier souligne les critiques sévères à l'égard de la Révolution de Juillet contenues dans les Lettres sur Paris, série d'articles commandés par Le Voleur alors que Balzac était à Tours, mais ne signale pas la quasi-absence de la figure du roi-citoyen dans l'œuvre romanesque de Balzac. En effet, Balzac avait choisi de décrire l'ambiance de l'époque louis-philipparde en relevant minutieusement les travers de la bourgeoisie montante et de l'aristocratie en déclin plutôt que d'analyser la personnalité de son monarque, qui n'offrait, pouvons-nous le supposer, aucun intérêt littéraire. L'ensemble des élus du système représentatif de la nouvelle monarchie constitutionnelle, que Balzac comparait à une véritable gérontocratie, reproduisait, selon lui, les bassesses de la cour : «Voici bientôt vingt ans d'essais constitutionnels, et nul ministre, soit venu de l'opposition, soit créé par le principe électif, n'a su régénérer l'administration. Ils ont tous eu le génie de recourir à l'emprunt; pas une voix n'a tonné, dans ces Chambres molles, contre ce suicide national, si niaisement continué par une série de vieillards successivement portés au ministère » (1956, p. 92).

Bref, ce chapitre brosse un tableau très général de l'époque louisphilipparde et se lit, au prix de quelques licences (raccourcis et omissions), comme un roman. Les bouleversements politiques et les progrès sociaux, technologiques et scientifiques qui y sont décrits préparent le terrain pour les deux chapitres suivants, consacrés à l'évolution de la pensée politique de Balzac, forcément influencée, comme nous le dit Métadier, par un « avenir sans visibilité » (p. 78).

\section{Description de Balzac en légitimiste non orthodoxe}

Les deux chapitres suivants, qui pourraient facilement être regroupés en un seul, étudient la pensée politique de Balzac in praesentia dans sa correspondance, ses articles et son œuvre romanesque. Faisant preuve d'une spontanéité rare, Métadier ne s'encombre pas d'un appareil 
Geneviève MADORE, «Balzac, homme politique légitimiste et visionnaire », @nalyses, automne 2007

méthodologique complexe - ni d'une surcharge bibliographique et procède au gré de ses réminiscences, dans un style qui rappelle celui de Philippe Muray (1984), en beaucoup plus épuré. Le chapitre s'écarte parfois de son sujet premier en effleurant aussi, de manière parfois décousue, l'importance grandissante de la science, l'émergence des utopistes ou autres "réformateurs modernes » et la popularité du mysticisme, trois sujets qui auraient pu être occultés car trop vastes pour être abordés en quelques lignes. On pardonnera néanmoins volontiers à l'auteur son style éclectique (qui cache, on le soupçonne, une érudition sous-jacente), même si certains passages laissent souvent le lecteur en plan, comme c'est le cas, par exemple, pour cette phrase qui se voulait une introduction à une description qui n'aura pas lieu «Il est aisé de suivre l'évolution des positions de Balzac à travers ses manifestes les plus significatifs. » (p. 104) - et le lien un peu facile et insuffisamment étayé entre le premier titre de La Comédie bumaine, Études sociales, et les velléités politiques de Balzac (p. 57).

L'argumentation du premier de ces deux chapitres, intitulé «Une pensée politique constante», repose sur des citations glanées essentiellement dans les articles dits « légitimistes» de Balzac (« Lettres sur Paris », «La politique des deux ministères », «Sur le gouvernement moderne [sic]», "Catéchisme social»). Fidèle à la doxa la plus répandue au sein de la critique balzacienne, Métadier dresse les contours d'un Balzac admirateur à la fois de Catherine de Médicis, de Robespierre et de Napoléon, qui exècre la tentation égalitaire et décrit la société comme une fade mosaïque. Balzac plaide pour un retour à l'ordre (naturel) qui s'appuie sur la raison d'État et sur l'ascension des seules «capacités» dans l'échelle sociale. Par conséquent, la «vision cohérente» que propose Métadier est celle d'un Balzac centralisateur dont la "pensée politique sera constamment occupée par une légitimité du pouvoir » (p. 95-96).

Prouvant en cela que le biographe n'est jamais bien loin, Métadier fait une revue de la genèse balzacienne et de ses influences. Cette parenthèse introductive au chapitre «Une pensée politique constante » s'attarde plus particulièrement au milieu familial : son père, disciple des Lumières et auteur de plusieurs "monographies», compta parmi ses 
Geneviève MADORE, «Balzac, homme politique légitimiste et visionnaire », @nalyses, automne 2007

premières influences; sa mère, lectrice de Swedenborg, lui légua sa curiosité pour le mysticisme. Parmi les premières influences figure aussi le collège de Vendôme, où le jeune Balzac s'imprégna d'histoire et de culture classique. À cette époque, la figure de Napoléon et «la nostalgie de la geste épique qui a bercé son enfance » (p. 63) entrèrent dans son imaginaire pour ne plus jamais en sortir. Parmi les influences féminines, déterminantes selon Métadier, se profilent Madame de Berny, qui «lui apporte le vécu de l'Histoire» (p. 66), la démocrate Zulma Carraud, la petite bourgeoise Louise Breugnot. On aurait aimé que l'auteur s'attarde davantage sur « l'idylle intellectuelle » qu'entretint Balzac avec George Sand, fervente socialiste, qui lui fit connaitre le terroir et ses habitants. Prisonnière en quelque sorte du régime tsariste de Nicolas $1^{\text {er }}$, Mme Hanska eut également un impact non négligeable sur la pensée politique de Balzac: «Cette confrontation avec le pouvoir absolu ne put que le conforter dans son opposition au retour en France d'un passé révolu » (p. 77). Balzac aurait même rédigé les passages dits monarchistes de l'«Avant-propos» en pensant l'«Étrangère », disposition d'esprit qui, pour Métadier, suffit à éloigner son auteur des ultras purs et durs.

Habilement, Métadier nous convainc - et c'est ce qui constitue la force de son essai — que Balzac fut un homme de son temps : son opinion ne différait pas tant de l'article contre la souveraineté populaire publié en 1838 dans la Revue des Deux Mondes. Certes, Balzac est encore loin du socialiste qu'une certaine critique avait cru discerner; il y a loin de lui à Blanqui. En ce sens, il est difficile d'être d'accord avec l'idée que «En 1824, Balzac entrevoit la lutte des classes » (p. 79), affirmation que Métadier illustre en prenant pour exemple le Code des gens honnêtes, qui, selon nous, est davantage une «monographie» sur l'escroquerie qu'un manifeste socialiste. Par contre, une énumération des œuvres de jeunesse prouve l'existence d'un jeune Balzac proche de la révolution (Cromwell et Jean-Lonis). Métadier souligne aussi avec justesse l'ambivalence politique des Chouans, le républicanisme du Colonel Chabert et mentionne au passage Les Lettres sur Paris, La Peau de chagrin pour leur critique de la France immédiatement après la Révolution de Juillet, La Duchesse de Langeais et 
Geneviève MADORE, «Balzac, homme politique légitimiste et visionnaire », @nalyses, automne 2007

Les Contes drolatiques pour leur critique de l'aristocratie et de l'Ancien Régime.

Toujours dans le cadre de cette réhabilitation politique de Balzac, Métadier aborde de front une question controversée : celle de son «apparent retournement» (p. 85), en 1832, vers le parti légitimiste : «En se proclamant royaliste comme à regret après la sévérité de son jugement sur Charles $\mathrm{X}$ et la virulence de ses diatribes contre Louis-Philippe, Balzac s'est sans doute souvent senti mal à l'aise » (p. 91). De fait, il n'est pas sans pertinence de nuancer la thèse du Balzac légitimiste: «En dépit de son adhésion de 1832 au parti royaliste, il n'a jamais été possible de voir en Balzac un apôtre de l'Ancien Régime» (p. 144). Il souhaite plutôt l'avènement d'une monarchie encadrée d'institutions et des citoyens protégés en vertu d'un Code civil qui les rende égaux devant la loi.

Le point de vue mitigé de Balzac sur le peuple est soulevé dans le chapitre "Une pensée politique constante ». Contrairement à George Sand, Balzac se prononce contre le suffrage universel. "Pour l'élection, George Sand fait confiance au suffrage universel et refuse l'élitisme du mérite ou des capacités cher à Balzac» (p. 133). Il faut tout de même préciser que "récuser le suffrage universel ne signifie pas pour Balzac récuser l'élection qui est nécessaire pour faire émerger les capacités» (p. 97). La menace populaire, ce «Robespierre à une tête et à vingt millions de bras » (1970, p. 61), pourrait être contenue par la religion et un pouvoir autoritaire. Craignant, comme bon nombre de ses contemporains, un retour de 1793, Balzac est néanmoins sensible au sort des masses, surtout à la situation économique précaire de la paysannerie. C'est pourquoi, selon Métadier, l'essentiel de sa pensée sociale tiendrait dans cet extrait de l'«Essai du parti royaliste » : «La meilleure société doit donc être celle qui, tout en donnant du pain aux prolétaires, en leur offrant les moyens nécessaires pour s'instruire et posséder, contraint néanmoins les excès probables de la partie souffrante d'une nation en présence de la partie aisée ou riche» (1956, p. 81). En somme, Balzac aurait donc été légitimiste a contrario. Cette nuance a déjà été apportée par Alexandre Courteix dans son étude des liens entre l'œuvre balzacienne 
Geneviève MADORE, «Balzac, homme politique légitimiste et visionnaire », @nalyses, automne 2007

et la révolution de 1789: Balzac avait rapproché les idéologies républicaines et absolutistes par leur conception unitaire du pouvoir (1997, p. 145). Pour éviter que le pouvoir des intérêts, de plus en plus menaçant sous la Monarchie de Juillet, empiète sur celui des capacités, il était préférable de conserver au pouvoir un parti centralisateur.

Le chapitre suivant, intitulé "Transparence politique de l'œuvre", s'articule autour de la pensée politique de Balzac dans la décennie 1840 et s'intéresse surtout à son œuvre romanesque. La France de 1840 est en plein crise : « grève générale, dissolution de la Chambre, démissions répétées des gouvernements" (p. 135-136). L'œuvre de Balzac s'obscurcit (Le Cousin Pons, La Cousine Bette, La Rabouilleuse). Il est ici tentant de mettre en parallèle Métadier avec d'autres critiques éminents qui, eux aussi, ont donné une importance particulière à l'année 1840. Curieusement, Nicole Mozet affirme qu'à cette époque s'effectue un tournant esthétique et idéologique: «l'acceptation tardive du phénomène révolutionnaire » et de la marche vers l'égalité, conjuguée à une "réorientation du roman vers la problématique de la conquête agressive de la bourgeoisie au pouvoir» (1990, p. 6). Courteix, quant à lui, affirme que Balzac renouera, à cette époque, avec la politique bonaldienne (1997, p. 65). Métadier nous apprend qu'en 1842, un vieux républicain du nom de Hetzel entreprend la publication des œuvres complètes de Balzac (p. 141). Il insiste d'ailleurs sur l'appui que Balzac a donné à la République lors des événements de 1848, sans doute parce que c'était, selon lui, la seule solution possible.

Pour Métadier, Illusions perdues serait le roman le plus «transparent» de Balzac, car il dépeint un monde où le Cénacle s'oppose à la presse parisienne, le savant à la petite aristocratie provinciale, et met en scène le personnage de Michel Chrestien, jeune républicain à l'âme pure. Ces thèmes constituent néanmoins la preuve que l'envergure de ce roman tient à bien plus qu'à sa seule portée politique. Contrairement à ce qu'en pense Métadier, il me semble impossible de voir les articles «Lettre sur le travail», qui s'oppose à la coalition ouvrière, et "Catéchisme social», inédit sur l'inégalité naturelle entre les êtres humains, deux textes fondés sur un argumentaire scientifique factice, 
Geneviève MADORE, «Balzac, homme politique légitimiste et visionnaire », @nalyses, automne 2007

comme des textes révolutionnaires. Certes, Balzac était sensible à l'importance du travail pour la bonne marche de la société et admettait l'inutilité des privilégiés sur le plan économique : "la notion du travail, œuvre essentielle de l'homme, fait partie de son credo politique » (p. 139). À ce sujet, l'auteur aurait pu nous renvoyer plutôt aux articles de Balzac sur le métier d'écrivain publiés dans les années 1830. Bien loin d'avoir emprunté un tournant socialiste, il nous semble que la pensée politique balzacienne, de plus en plus légitimiste, s'est rigidifiée. Il faut toutefois s'accorder avec Métadier pour ne pas faire de l'humanisme la chasse gardée du socialisme: «Dans son tableau de la société, c'est le paupérisme en général qui s'impose à son observation au regard du règne de l'argent aux mains d'une bourgeoisie avide et sans scrupule qu'il juge si sévèrement, "l'opinion bourgeoise confond les classes laborieuses et les classes dangereuses, le prolétariat et la pègre, la misère et le crime"» (p. 152). Enfin, on saura toujours reconnaitre chez Balzac un sens du pragmatisme et la grande acuité de son regard sur l'argent et ses mécanismes : «L'argent, il en connait tous les cheminements, l'âpreté au gain, l'avarice, la malhonnêteté, la procédure » (p. 152).

\section{«La clairvoyance de Balzac »}

Dans son Art romantique, Baudelaire tient ces propos: «J'ai maintes fois été étonné que la grande gloire de Balzac fût de passer pour un observateur; il m'avait toujours semblé que son principal mérite était d'être visionnaire, et visionnaire passionné » (1968, p. 252). L'auteur des Fleurs du mal admirait cette verve avec laquelle Balzac dépeignait, plus vrais que nature, des personnages «depuis le sommet de l'aristocratie jusqu'aux bas-fonds de la plèbe » (p. 252). Dans cette même veine, dans le dernier chapitre, intitulé «La clairvoyance de Balzac», Métadier dénonce les stéréotypes, ces «faux semblants frisant le ridicule» (p. 163), rattachés à la personne de Balzac : boulimique, chineur, obsédé par l'argent. Qu'on se le tienne pour dit:Balzac était plutôt un homme sédentaire et un fin connaisseur des mœurs de son époque. Dès 1829, dans la Physiologie du mariage, il se montre en faveur de l'émancipation des femmes en dénonçant l'institution du mariage. En 1841, dans Le Curé de village, 
Geneviève MADORE, «Balzac, homme politique légitimiste et visionnaire », @nalyses, automne 2007

dans le cadre d'une diatribe contre l'institution juridique, il s'oppose en toutes lettres à la peine de mort, faisant écho au Dernier jour d'un condamné (1829) de Victor Hugo. Il n'est pas surprenant qu'il « [soit] devenu le censeur impitoyable de la monarchie philiparde [sic], en fustigeant dans ses cuvres la bourgeoisie, avide de faveurs et d'argent» (p. 177-178), mais Métadier n'étaye pas suffisamment sa thèse pour nous convaincre que Balzac a été un précurseur.

Balzac croyait que les «capacités » pouvaient régénérer la société : ce leitmotiv de l'essai de Métadier dévoilerait un Balzac plus proche de la sociologie que de la politique. Balzac a souvent déploré que des savants de naissance modeste comme David Séchard (Illusions perdues) aient été condamnés à rester dans l'ombre: «Dans Louis Lambert, qui emprunte tant à l'autobiographie de son auteur, Balzac nous dit: "la force est l'unique loi de l'homme"» (p. 172). Cette ligne directrice de la pensée politique balzacienne sur laquelle insiste Métadier est moins la manifestation d'un esprit visionnaire que la réflexion typique d'un homme du début du XIX ${ }^{\mathrm{e}}$ siècle devant la disparition des privilèges et l'apparition du «Enrichissez-vous!» de Guizot: «la constante de la pensée politique de Balzac: le juste-milieu conservateur de la bourgeoisie qui oublie l'homme où réside la force de la société » (p. 175). Parler de marginalité serait davantage conforme au personnage de Balzac: «L'immense mérite de Balzac est d'avoir eu le courage d'aller à contre-courant des dérives de la société où il était entré de force et qui l'accueillait et le rejetait tout à la fois. Il en a dénoncé sans complaisance toutes les tares» (p. 172-173). En effet, osons-nous dire, malgré l'ajout de la particule à son patronyme, Balzac n'eut jamais officiellement accès à l'aristocratie, qu'il a d'ailleurs vivement critiquée dans son œuvre, ni à cette bourgeoisie prospère, dont il dénonce les penchants matérialistes. Il fut même en marge des principales instances littéraires de son époque; celui qui a défendu le statut de l'artiste, notamment dans «La lettre adressée aux écrivains français du XIX ${ }^{\mathrm{e}}$ siècle », a été tenu en dehors du Cénacle.

En somme, la démonstration de Métadier, plus descriptive qu'analytique et dont on a peine à saisir la structure profonde, ne nous convainc pas de l'existence d'un Balzac socialisant, mais réussit à bien 
Geneviève MADORE, «Balzac, homme politique légitimiste et visionnaire», @nalyses, automne 2007

étayer la thèse du Balzac légitimiste modéré qui a peine à trouver sa place dans une société en constant bouleversement. En ce sens, il mène à bien un de ses objectifs, qui était de sortir des sentiers battus. On peut regretter que le chapitre sur la France de Louis-Philippe ne fasse pas ressortir de manière substantielle des événements qui n'auraient pas encore été évoqués par des historiens ou des littéraires, ainsi que le promet l'introduction. Enfin, on peut déplorer quelques inexactitudes et citations mal sorties de leur contexte, telles celle tirée des Paysans (p. 152) ou l'allusion au Curé de village (p. 117). Ces quelques manquements ne compromettent en rien, cependant, l'intérêt que pourront porter au livre de Métadier les balzaciens rompus à la lecture de la critique, et qui verront cet essai comme une synthèse érudite sur une des multiples facettes de leur auteur de prédilection. Le néophyte, quant à lui, appréciera cet ouvrage accessible — et succinct - et y trouvera des pistes de recherche prometteuses. Devant la difficulté du sujet, Métadier a bien su relever le pari de la simplicité et de l'éclectisme.

Conscient de ces limites épistémologiques - comme il l'admet dans son prologue -Métadier a l'immense mérite de jeter un œil candide sur son sujet, en plus d'insister sur l'humanisme de Balzac, un trait qui n'entre pas nécessairement en contradiction avec un élitisme avoué, sur lequel la critique insiste trop souvent. Balzac, et en cela réside peut-être sa "clairvoyance», avait affirmé la supériorité de l'écrivain sur l'homme d'État: «La loi de l'écrivain, ce qui le fait tel, ce qui, je ne crains pas de le dire, le rend égal et peut-être supérieur à l'homme d'État, est une décision quelconque sur les choses humaines, un dévouement absolu à des principes» (1976, p. 12). Philosophe, l'auteur clôt son livre sur cette citation de Maurice Barrès: «Pas la peine de discuter pour savoir si Balzac était socialiste ou réactionnaire, la pensée de Balzac embrasse plus de choses qu'un vocabulaire n'en exprime, il faut écouter ce génie comme on se promène dans la forêt » (p. 177).

Référence: Paul Métadier, Balzac homme politique, Paris, L'Harmattan, coll. «Espaces littéraires », 2006, 194 p. 


\section{Bibliographie}

BAlZAC, Honoré de. 1976 [1842], «Avant-propos », La Comédie humaine, Paris, Gallimard, coll. «Bibliothèque de la Pléiade », t. I, p. 7-20;

—. 1970 [1844], Les Paysans, Paris, Garnier-Flammarion;

—. 1956 [inédit, 1830], «Du gouvernement moderne », Euvres complètes, Paris, Club de l'honnête homme, vol. 23, p. 88-102;

—. 1956 [1832], « Essai sur la situation du parti royaliste », CEuvres complètes, Paris, Club de l'honnête homme, vol. 23, p. 72-88.

BAUdelaire, Charles. 1968 [1869], L'Art romantique, Paris,

Garnier-Flammarion.

COURTEIX, Alexandre. 1997, Balzac et la Révolution française. Aspects idéologiques et politiques, Paris, PUF.

Mozet, Nicole. 1990, Balzac au pluriel, Paris, PUF.

MURAY, Philippe. 1984, Le $19^{e}$ siècle à travers les âges, Paris, Denoël.

Schuerewegen, Franc. 2004, Balzac, suite et fin, Lyon, Presses de l'École normale supérieure, coll. « signes ». 